\title{
Sequential Sampling to Assess the Incidence of Infection by Monilinia vaccinii-corymbosi in Mechanically Harvested Rabbiteye Blueberry Fruit
}

\author{
W. E. Copes, H. Scherm, and G. O. Ware
}

First author: USDA-ARS, Small Fruit Research Station, Poplarville, MS 39470; second author: Department of Plant Pathology, University of Georgia, Athens 30602; and third author: Experimental Statistics, College of Agricultural and Environmental Sciences, University of Georgia, Athens 30602.

Accepted for publication 7 December 2000.

\begin{abstract}
Copes, W. E., Scherm, H., and Ware, G. O. 2001. Sequential sampling to assess the incidence of infection by Monilinia vaccinii-corymbosi in mechanically harvested rabbiteye blueberry fruit. Phytopathology 91:348-353.

Blueberry fruit infected by Monilinia vaccinii-corymbosi, the causal agent of mummy berry disease, are unsuitable for use in processed food products. Fruit shipments that exceed a disease incidence threshold of $0.5 \%$ are redirected to alternative markets with substantial reductions in economic return to the producer. Because of this low tolerance, a sampling procedure with defined statistical properties is needed to determine disease incidence in the packinghouse. In this study, a sequential sampling plan was developed based on counts and dispersion patterns of infected fruit in 23 loads of mechanically harvested rabbiteye blueberries. Each load was sampled 20 to 100 times, with each sample contain-

computed, all of which suggested aggregation of infected fruit. Because $k$ was variable across loads, Iwao's regression procedure, which does not assume a single frequency distribution with fixed parameters describing the counts of infected fruit, was used to develop upper and lower stop lines for sequential sampling. For $\alpha=0.05$ and assuming a total of 250 fruit per $550-\mathrm{cm}^{3}$ sample, the resulting sampling plan would require only one sample to conclude that a load exceeds the threshold if the number of infected fruit in that sample is greater than four. A minimum of six samples would be needed to conclude that disease incidence in a load is below the threshold if the cumulative total of infected fruit in these samples is zero. Resampling analysis showed that most fruit loads could be classified reliably with $<10$ samples per load; for loads with a disease incidence very close to the $0.5 \%$ threshold, $<50$ samples were needed on average. Stop lines for sequential sampling for different fruit size classes are presented.
\end{abstract} ing $550 \mathrm{~cm}^{3}$ of fruit. Various dispersion statistics ( $k$ of the negative binomial distribution, Lloyd's index of patchiness, and Iwao's $b$ ) were
Additional keywords: disease detection, Vaccinium ashei.
Mummy berry disease, caused by the discomycete Monilinia vaccinii-corymbosi (Reade) Honey, is the most costly disease of rabbiteye blueberry (Vaccinium ashei Reade), the dominant blueberry species grown in Georgia (21). Losses in the field include a reduction in total fruit volume due to the loss of fruiting wood in the spring from shoot and flower blight, and from infected fruit that mummify and drop to the ground before or at harvest $(2,8)$. Greater losses can occur after harvest when a fruit shipment is downgraded due to an excessive incidence of infected fruit. This is particularly important for fruit loads intended for use in processed food products, for which a tolerance of $0.5 \%$ disease incidence has been established by marketing cooperatives (W. E. Copes, unpublished data). Most rabbiteye blueberries are harvested mechanically, washed and graded, and freeze-dried for use in packaged snack foods and cooking products. The standard sorting and grading process in the packinghouse, which comprises a series of fans, screens, wash tanks, and a visual inspection area (1), is only partly effective in removing infected fruit from the product intended for freeze-drying $(\mathrm{H}$. Scherm and W. E. Copes, unpublished data). Once dried, fruit infected by $M$. vacciniicorymbosi harden, thus becoming unsuitable for use in these products.

Corresponding author: W. Copes; E-mail address: wcopes@ars.usda.gov

Publication no. P-2001-0118-02R

This article is in the public domain and not copyrightable. It may be freely reprinted with customary crediting of the source. The American Phytopathological Society, 2001.
Because of the economic losses associated with blueberry loads that exceed the mummy berry tolerance and the inability of current grading practices to remove all infected fruit, a reliable procedure is needed to quantify disease incidence under commercial conditions. Since 1994, packinghouse operators in Georgia have used a procedure in which a single $952-\mathrm{cm}^{3}$ fruit sample is processed in a blender and physically examined for the presence of infected fruit. Although this method can detect infected fruit, its accuracy and precision have been found inadequate in a recent evaluation (20). As an alternative, Scherm and Copes (20) described the use of symptomatology for quantifying mummy berry incidence. Although the latter assessment method was considerably more accurate and precise than the previously recommended blender method (20), the low threshold for infected fruit mandates a sampling protocol that makes acceptance or rejection decisions with defined statistical properties. To date, no such sampling strategy is available for quantifying mummy berry incidence in blueberry packinghouses.

Most sampling plans for disease incidence are based on fixedprecision sampling in which a predetermined number of samples is collected so as not to exceed a given confidence interval or coefficient of variation around the true mean (11). This sampling strategy generally requires large numbers of samples to achieve moderate to high levels of precision. However, when making acceptance-rejection decisions similar to those involved in assessing blueberry loads for the incidence of fruit infected by $M$. vacciniicorymbosi, fewer samples may be sufficient to decide reliably whether disease incidence is above or below the threshold. Sequential sampling, an alternative to fixed-precision sampling, is 
based on the premise that for acceptance-rejection decisions, high levels of precision are important only if disease incidence is close to the threshold. By contrast, in cases where disease incidence is sufficiently greater or lower than the threshold, a correct classification can be reached with less precision (and thus smaller numbers of samples). Sequential sampling has been used extensively for decision making in entomology $(3,16,18,22,25)$, but to a much lesser extent in plant pathology $(5,14,19,23)$.

The standard approach for developing sequential sampling plans, referred to as sequential probability ratio test or Wald's procedure, involves the fitting of frequency count data to a suitable frequency distribution (such as the binomial, negative binomial, or Poisson) and use of the resulting parameter estimates in calculating stop lines $(4,24,25)$. A limitation of this approach is the need for a single frequency distribution with fixed parameter values describing all populations to which the sequential sampling plan is to be applied. As an alternative, Iwao $(9,10)$ developed an empirical procedure that adapts to variability in frequency distributions among populations, based on the regression of Lloyd's index of mean crowding, $\stackrel{*}{x}$, on the mean, $\bar{x}$, followed by the calculation of stop lines using the estimated regression parameters. Iwao's procedure has not previously been used for sequential sampling plan development in plant pathology.

The objective of this study was to determine dispersion patterns of fruit infected by $M$. vaccinii-corymbosi in loads of mechanically harvested blueberries in commercial packinghouses by intensive sampling of fruit loads with a wide range of disease incidence levels. This information was then used to derive a sequential sampling plan based on Iwao's procedure. The resulting sampling plan was further evaluated by resampling from packinghouse data sets to arrive at average performance values and associated variances.

\section{MATERIALS AND METHODS}

Sampling procedure. Six packinghouses in four counties in southeastern Georgia were surveyed in June and July 1997 and 1998 to determine the incidence of fruit infected by M. vacciniicorymbosi in blueberry loads that had been mechanically harvested into plastic trays, stacked onto pallets, and transported to the packinghouses. A total of 23 fruit loads from various farms, cultivars, and harvest dates were assessed (Table 1). Each load consisted of five stacks of trays, each having a volume of $\approx 40$ liters, with typically 6 to 10 trays per stack. From each load, 20 (1997) or 80 to 100 (1998) $550-\mathrm{cm}^{3}$ fruit samples were drawn, with no more than two samples taken from the same tray. The number of infected fruit in each sample was determined visually as described previously (20). All fruit exhibiting symptoms of mummy berry disease or other abnormalities were cross-sectioned to verify the presence of mycelium or entostroma typical of $M$. vacciniicorymbosi. Total numbers of fruit were counted for three $550-\mathrm{cm}^{3}$ samples per load.

Descriptive statistics. Means $(\bar{x})$ and variances $\left(s_{x}^{2}\right)$ of mummy berry incidence across the samples were calculated for each load. Three dispersion statistics, the parameter $k$ of the negative binomial distribution, Lloyd's index of mean crowding ( $\left.x_{x}^{*}\right)$, and Lloyd's index of patchiness (LIP) were also computed for each load. $k$ was estimated with software provided in Pedigo and Zeiss (18). Large values for $k$ indicate a random distribution, whereas $k$ values approaching zero indicate increasing aggregation (6). Lloyd's index of mean crowding and Lloyd's index of patchiness were calculated as $\dot{x}=\bar{x}+s_{x}^{2} / \bar{x}-1$ and LIP $=\dot{x} / \bar{x}$, respectively. The latter index indicates how "crowded" an infected fruit is in a given sample relative to one with a random distribution of infected fruit (6). Following Iwao (9), $x$ was regressed against $\bar{x}$ across all loads as

$$
\stackrel{*}{x}=a+b \times \bar{x}
$$

where $a$ and $b$ are parameters. The dispersion pattern of infected fruit is regular, random, and aggregated for $b$ values less than, equal to, and greater than one, respectively $(6,9,22)$.

Sequential sampling plan. In the present study, most fruit loads were described adequately by the negative binomial distribution, but $k$ varied by more than 1 order of magnitude across the loads (Table 1). Thus, Iwao's procedure $(9,10)$, which does not assume an underlying theoretical distribution that fits all loads, was used for sampling plan development. Upper $\left(T_{u}\right)$ and lower $\left(T_{l}\right)$ stop limits for sequential sampling were calculate $\mathrm{d}$ as

$$
T_{u}=n_{s} \times x_{0}+z_{a / 2} \times \sqrt{n_{s}\left[(a+1) x_{0}+(b-1) x_{0}^{2}\right]}
$$

and

$$
T_{l}=n_{s} \times x_{0}-z_{a / 2} \times \sqrt{n_{s}\left[(a+1) x_{0}+(b-1) x_{0}^{2}\right]}
$$

where $n_{s}$ is the cumulative number of samples, $x_{0}$ is the tolerance threshold (1.25 infected fruit per 550- $\mathrm{cm}^{3}$ sample), and $a$ and $b$ are, respectively, the intercept and slope of Iwao's regression (equation 1) $(10,22) . z_{\alpha / 2}$, the value of the standard normal variate at significance level $\alpha / 2$, is a parameter defining the distance between the two stop lines (4).

With sequential sampling, an assessor continues sampling until the cumulative number of infected fruit exceeds $T_{u}$ (at which time disease incidence in the load is classified as exceeding the threshold) or drops below $T_{l}$ (at which time the load is classified as below the threshold). The maximum number of samples, $n_{\max }$, that needs to be collected if the cumulative number of infected fruit stays between $T_{u}$ and $T_{l}$ was calculated as

$$
n_{\max }=z_{\alpha / 2}^{2} / D^{2}\left[(a+1) / x_{0}+b-1\right]
$$

$(10,26)$, where $D$ is the desired precision (expressed as a proportion between 0 and 1). If the cumulative number of infected fruit is still between $T_{u}$ and $T_{l}$ at $n_{\max }$, it can be concluded, with precision $D$, that disease incidence is not significantly different from the threshold.

Initially, $T_{u}, T_{l}$, and $n_{\max }$ were calculated assuming a "typical" blueberry load with 250 fruit per $550-\mathrm{cm}^{3}$ sample, resulting in $x_{0}=1.25$. To account for loads with different numbers of fruit per sample due to variations in fruit size, the calculations were repeated for fruit number classes ranging from 200 to 400 fruit per sample. Variations in fruit numbers per sample were implemented by changing $x_{0}$. For example, based on the commercial tolerance of $0.5 \%$ disease incidence, $x_{0}$ was set at 1.0 and 2.0 for samples containing 200 and 400 fruit, respectively. The computations used common $a$ and $b$ parameters for all fruit number classes after initial calculations showed limited sensitivity of $T_{u}$ and $T_{l}$ to variations in $a$ and $b$ similar to those observed when Iwao's regression (equation 1) was computed separately for fruit loads with low versus high numbers of fruit per sample (data not shown).

Resampling analysis. Analyses were carried out to determine the number of samples an assessor would have to draw from each of the fruit loads in Table 1, using the sequential sampling plan defined by equations 2 through 4 , before being able to decide whether disease incidence was above or below the threshold. SAS code (SAS Institute Inc., Cary, NC) was written that resampled the array of counts of infected fruit from each load randomly and without replacement until the cumulative number of infected fruit either exceeded $T_{u}$, dropped below $T_{l}$, or until $n_{\max }$ (calculated with $D=0.25$ ) was reached. Five-hundred resampling runs were carried out for each load to calculate the mean and standard deviation of the sample number at which the stop limit was reached. All calculations used $x_{0}$ values corrected for differences in fruit numbers per load as described previously. 


\section{RESULTS AND DISCUSSION}

Descriptive statistics. The mean number of fruit per $550-\mathrm{cm}^{3}$ sample ranged from 206 to 373 (Table 1). Fruit loads assessed in 1998 contained more fruit per sample than those assessed in 1997 (Fig. 1), most likely because drought conditions in 1998 resulted in smaller fruit sizes. For both years combined, the most frequent fruit number class had a midpoint of 250 fruit per sample (Fig. 1). Thus, a typical blueberry load was considered to contain 250 fruit per sample in subsequent calculations.

Mean mummy berry incidence in the loads ranged from 0.05 to 12.4 infected fruit per sample, with 10 loads exceeding the threshold of $0.5 \%$ (Table 1). When frequency distributions were fitted to the 1997 data sets (20 samples per load), $k$ of the negative binomial distribution could not be computed for loads with low disease incidence because too few samples contained infected fruit (Table 1); these loads were best described by a Poisson distribution, indicative of a random dispersion pattern (data not shown). With 80 to 100 samples per load (1998 data sets), all loads, including those with very low disease incidence, were well described by the negative binomial distribution (Table 1). For these loads, parameter estimates for $k$, which ranged from 0.607 to 7.16 (Table 1), generally indicated aggregation of infected fruit. Because $k$ varied by more than 1 order of magnitude, Iwao's procedure $(9,10)$ was selected for subsequent sampling plan development. The observation that $k$ varies with the mean is well supported by the literature on dispersion patterns of plant diseases $(6,13,19)$; nevertheless, investigators often tacitly assume such parameters to be constant when developing sampling plans.

Lloyd's index of mean crowding, $x$, and Lloyd's index of patchiness, LIP, also indicated that infected fruit tended to be aggregated (Table 1). This was supported by Iwao's regression (equation 1), which resulted in the equation $\dot{x}=0.343+1.27 \bar{x}(P<0.0001$; $\left.r^{2}=0.927\right)($ Fig. 2) with a slope ( $b$ parameter) significantly greater than one. When regression lines were fitted for the 2 years separately and tested for heterogeneity of slopes (SAS Institute), they were not significantly different $(b=1.26$ and 1.40 for 1997 and 1998 , respectively; $P=0.5969$ ).

The observation that infected fruit in packinghouse samples tended to be aggregated is not surprising. Such a pattern could arise when harvesting from fields in which disease is aggregated, using a harvesting and handling process that allows only limited mixing of fruit. Several aspects of the mummy berry pathosystem favor disease aggregation in the field. These include aggregation of initial inoculum, e.g., due to differences in soil moisture favorability for carpogenic germination (12) along with limited disease spread because shoot infection (by ascospores produced in apothecia) and fruit infection (by conidia produced on blighted

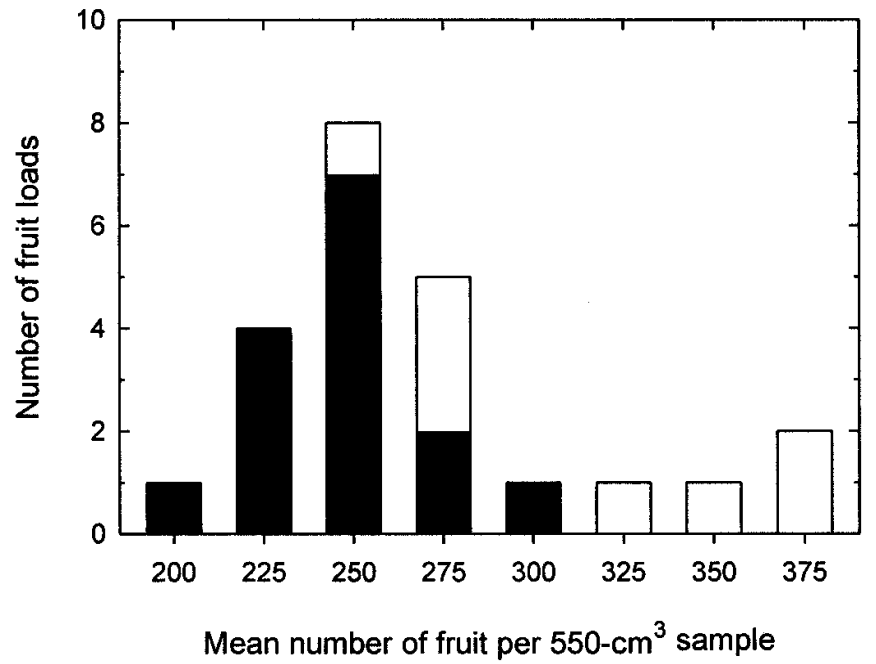

Fig. 1. Frequency distribution of the total number of fruit per sample in loads of mechanically harvested rabbiteye blueberries from commercial packinghouses in southeastern Georgia in 1997 (匹) and 1998 ( $\square$ ). Fruit numbers based on the mean of three $550-\mathrm{cm}^{3}$ samples per load.

TABLE 1. Incidence and dispersion statistics of fruit infected by Monilinia vaccinii-corymbosi in samples of mechanically harvested rabbiteye blueberries from commercial packinghouses in southeastern Georgia in 1997 and 1998

\begin{tabular}{|c|c|c|c|c|c|c|c|c|}
\hline \multirow[b]{2}{*}{ Fruit load } & \multirow[b]{2}{*}{ No. of samples ${ }^{\mathrm{a}}$} & \multirow[b]{2}{*}{ Fruit/sample ${ }^{b}$} & \multicolumn{3}{|c|}{ Disease incidence } & \multicolumn{3}{|c|}{ Dispersion statistics ${ }^{\mathrm{c}}$} \\
\hline & & & Mean, $\bar{x}$ & Variance, $s_{x}^{2}$ & $\%$ & $k$ & Mean crowding, $\stackrel{*}{x}$ & LIP \\
\hline 1 & 20 & 233 & 0.05 & 0.05 & 0.022 & - & 0.050 & 1.00 \\
\hline 2 & 20 & 238 & 0.05 & 0.05 & 0.021 & - & 0.050 & 1.00 \\
\hline 4 & 20 & 256 & 0.20 & 0.16 & 0.078 & - & 0 & 0 \\
\hline 5 & 20 & 240 & 0.20 & 0.26 & 0.083 & - & 0.500 & 2.50 \\
\hline 6 & 80 & 246 & 0.29 & 0.40 & 0.12 & 0.902 & 0.669 & 2.31 \\
\hline 10 & 20 & 226 & 0.60 & 0.74 & 0.27 & 2.03 & 1.72 & 2.87 \\
\hline 11 & 80 & 373 & 0.73 & 1.5 & 0.20 & 0.607 & 0.833 & 1.14 \\
\hline 12 & 20 & 262 & 1.1 & 1.1 & 0.42 & - & 1.10 & 1.00 \\
\hline 13 & 80 & 332 & 1.4 & 2.8 & 0.42 & 1.06 & 2.42 & 1.73 \\
\hline 14 & 100 & 281 & 1.6 & 3.5 & 0.57 & 1.40 & 2.76 & 1.73 \\
\hline 15 & 80 & 368 & 1.9 & 4.3 & 0.52 & 1.68 & 3.16 & 1.66 \\
\hline 21 & 20 & 214 & 5.8 & 37.3 & 2.7 & 0.616 & 11.2 & 1.93 \\
\hline 22 & 20 & 229 & 6.4 & 24.5 & 2.8 & 1.69 & 9.21 & 1.44 \\
\hline 23 & 20 & 206 & 12.4 & 28.8 & 6.0 & 7.16 & 13.7 & 1.10 \\
\hline
\end{tabular}

a Each sample contains $550 \mathrm{~cm}^{3}$ of fruit.

${ }^{\mathrm{b}}$ Mean fruit count of three samples.

${ }^{\mathrm{c}} k$ denotes $k$ of negative binomial calculated by fitting the negative binomial distribution to frequency count data (number of samples with $0,1,2,3$, etc. infected fruit); Lloyd's index of mean crowding calculated as $x=\bar{x}+s_{x}^{2} / \bar{x}-1$; Lloyd's index of patchiness calculated as LIP $=x / x$; and - indicates data set not described adequately by the negative binomial distribution. 
shoots) are both monocyclic processes $(2,8)$. In addition, because fruit infection occurs only via the stigma and style of open flowers $(2,8)$, the tendency of bloom times to be more similar among flowers within individual flower clusters than among flower clusters within individual blueberry bushes may lead to withinbush aggregation of infected fruit. Mechanical blueberry harvesters pick fruit from single rows and deposit them directly into small ( $\approx 40$-liter) trays without subsequent combining of individual tray contents into larger units, thereby providing little opportunity for mixing of fruit collected from a larger area. The harvesting process thus favors the continued association of fruit from individual bushes after harvest, thereby preserving the dispersion pattern present in the field. This system differs from those in which a product is harvested mechanically and combined into larger volumes, thereby effectively mixing infected and healthy units and decreasing the aggregation of diseased units. Examples include

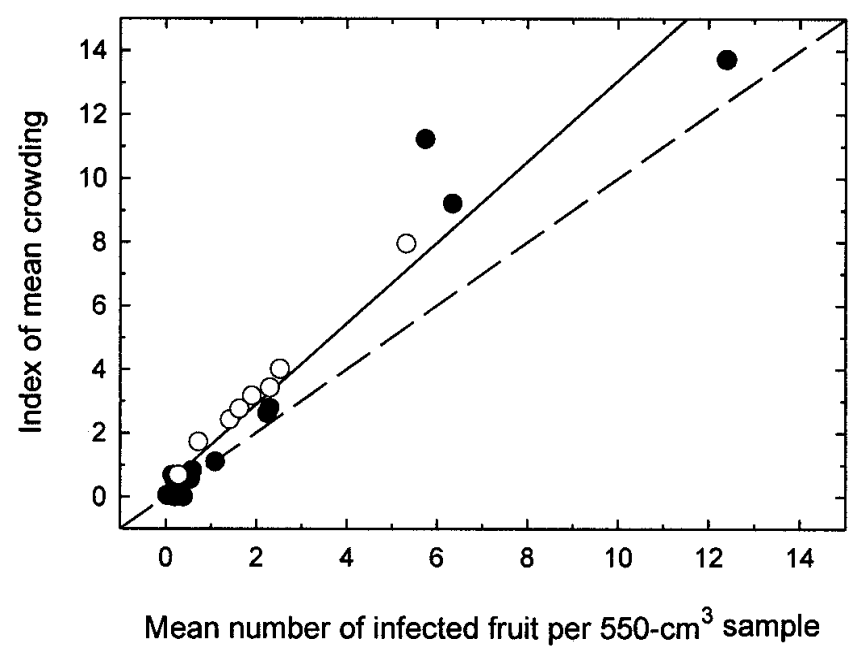

Fig. 2. Regression of Lloyd's index of mean crowding, $x$, on the mean number of fruit per sample infected by Monilinia vaccinii-corymbosi, $\bar{x}$, for loads of mechanically harvested rabbiteye blueberries assessed in commercial packinghouses in southeastern Georgia in $1997(\bullet)$ and $1998(\bigcirc)$. The regression equation (solid line) is $x=0.343+1.27 \bar{x} \quad\left(P<0.0001 ; r^{2}=0.927\right)$, with a slope significantly greater than that of the $1: 1$ line (dashed line).

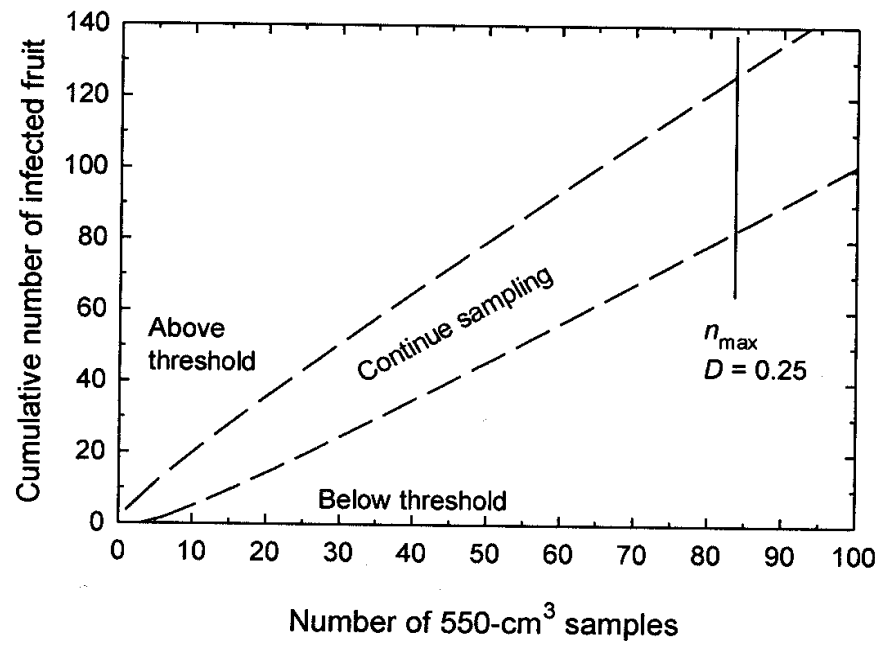

Fig. 3. Sequential sampling plan to determine whether the incidence of fruit infected by Monilinia vaccinii-corymbosi in loads of mechanically harvested rabbiteye blueberries is above or below a threshold of $0.5 \%$ (1.25 infected fruit, assuming a total of 250 fruit per sample). Stop lines generated with equations 2 and 3, with $\alpha=0.05$. The maximum number of samples, $n_{\max }$, that needs to be collected if the cumulative number of infected fruit stays between the two stop lines, was calculated with precision $D=0.25$ (equation 4). scab-infected wheat sampled from large truckloads (7) and gray mold-infected grape berries sampled from four gondola-trucks (15).

Sequential sampling plan. The sequential sampling plan to determine whether the incidence of infected fruit is above or below the threshold of $0.5 \%$ (1.25 infected fruit per sample, assuming a total of 250 fruit per sample) is shown in Figure 3. Only one sample would be needed to conclude that the load exceeds the threshold if the number of infected fruit in that sample is greater than four. In contrast, a minimum of six samples would be required before one could conclude that a load is below the threshold if the cumulative total of infected fruit in these samples is zero. Additional samples would be needed if the cumulative number of infected fruit stays between $T_{u}$ and $T_{l}$ as samples are being collected. The maximum number of samples, $n_{\max }$, at which one would stop sampling and conclude that disease incidence is not significantly different from the threshold is 83 for $D=0.25$ (Fig. 3).

Calculation of $T_{u}, T_{l}$, and $n_{\max }$ in Figure 3 was based on a typical blueberry load with a total of 250 fruit per $550-\mathrm{cm}^{3}$ sample (Fig. 1). However, the two stop limits were sensitive to fruit size, expressed as the total number of fruit per sample. Values of $T_{u}$ and $T_{l}$ at each sample increased as the total number of fruit per sample increased (Table 2). Because of the sensitivity of the sequential

TABLE 2. Sensitivity to fruit size, expressed as the total number of fruit per sample, of upper $\left(T_{u}\right)$ and lower $\left(T_{l}\right)$ stop limits of a sequential sampling plan to determine whether the incidence of fruit infected by Monilinia vacciniicorymbosi in loads of mechanically harvested rabbiteye blueberries is above or below a threshold of $0.5 \%$ a

\begin{tabular}{|c|c|c|c|c|c|c|c|c|c|c|}
\hline \multirow[b]{3}{*}{ No. } & \multicolumn{10}{|c|}{ No. of fruit per $550-\mathrm{cm}^{3}$ sample } \\
\hline & \multicolumn{2}{|c|}{$\begin{array}{c}200 \\
\left(x_{0}=1.0\right)\end{array}$} & \multicolumn{2}{|c|}{$\begin{array}{c}250 \\
\left(x_{0}=1.25\right) \\
\end{array}$} & \multicolumn{2}{|c|}{$\begin{array}{c}300 \\
\left(x_{0}=1.5\right)\end{array}$} & \multicolumn{2}{|c|}{$\begin{array}{c}350 \\
\left(x_{0}=1.75\right)\end{array}$} & \multicolumn{2}{|c|}{$\begin{array}{c}400 \\
\left(x_{0}=2.0\right)\end{array}$} \\
\hline & $T_{u}$ & $T_{l}$ & $T_{u}$ & $T_{l}$ & $T_{u}$ & $T_{l}$ & $T_{u}$ & $T_{l}$ & $T_{u}$ & $T_{l}$ \\
\hline 1 & 4 & - & 5 & - & 5 & - & 6 & - & 6 & - \\
\hline 2 & 6 & - & 7 & - & 8 & - & 9 & - & 10 & - \\
\hline 3 & 8 & - & 9 & - & 10 & - & 12 & - & 13 & - \\
\hline 4 & 9 & - & 11 & - & 13 & - & 14 & 0 & 16 & 0 \\
\hline 5 & 11 & - & 13 & - & 15 & 0 & 17 & 0 & 19 & 1 \\
\hline 6 & 13 & - & 15 & 0 & 17 & 1 & 19 & 1 & 22 & 2 \\
\hline 7 & 14 & 0 & 17 & 1 & 19 & 2 & 22 & 3 & 25 & 3 \\
\hline 8 & 16 & 0 & 19 & 1 & 21 & 3 & 24 & 4 & 27 & 5 \\
\hline 9 & 17 & 1 & 20 & 2 & 24 & 3 & 27 & 5 & 30 & 6 \\
\hline 10 & 18 & 2 & 22 & 3 & 26 & 4 & 29 & 6 & 33 & 7 \\
\hline 12 & 21 & 3 & 25 & 5 & 29 & 6 & 34 & 8 & 38 & 10 \\
\hline 14 & 24 & 4 & 29 & 6 & 33 & 9 & 38 & 11 & 43 & 13 \\
\hline 16 & 26 & 6 & 32 & 8 & 37 & 11 & 42 & 14 & 48 & 16 \\
\hline 18 & 29 & 7 & 35 & 10 & 41 & 13 & 47 & 16 & 53 & 19 \\
\hline 20 & 32 & 8 & 38 & 12 & 45 & 15 & 51 & 19 & 58 & 22 \\
\hline 24 & 37 & 11 & 44 & 16 & 52 & 20 & 60 & 24 & 67 & 29 \\
\hline 28 & 42 & 14 & 51 & 19 & 59 & 25 & 68 & 30 & 77 & 35 \\
\hline 32 & 47 & 17 & 57 & 23 & 66 & 30 & 76 & 36 & 86 & 42 \\
\hline 36 & 51 & 21 & 63 & 27 & 74 & 34 & 84 & 42 & 95 & 49 \\
\hline 40 & 56 & 24 & 68 & 32 & 81 & 39 & 93 & 47 & 105 & 55 \\
\hline 44 & 61 & 27 & 74 & 36 & 88 & 44 & 101 & 53 & 114 & 62 \\
\hline 48 & 66 & 30 & 80 & 40 & 94 & 50 & 109 & 59 & 123 & 69 \\
\hline 52 & 70 & 34 & 86 & 44 & 101 & 55 & 117 & 65 & 132 & 76 \\
\hline 56 & 75 & 37 & 92 & 48 & 108 & 60 & 125 & 71 & 141 & 83 \\
\hline 60 & 80 & 40 & 98 & 52 & 115 & 65 & 133 & 77 & - & - \\
\hline 64 & 84 & 44 & 103 & 57 & 122 & 70 & 140 & 84 & - & - \\
\hline 68 & 89 & 47 & 109 & 61 & 129 & 75 & - & - & - & - \\
\hline 72 & 94 & 50 & 114 & 65 & 135 & 81 & - & - & - & - \\
\hline 76 & 98 & 54 & 120 & 70 & - & - & - & - & - & - \\
\hline 80 & 103 & 57 & 126 & 74 & - & - & - & - & - & - \\
\hline 90 & 114 & 66 & - & - & - & - & - & - & - & - \\
\hline 100 & 125 & 75 & - & - & - & - & - & - & - & - \\
\hline
\end{tabular}

a Stop lines generated with equations 2 and 3 with $\alpha=0.05$.

${ }^{\mathrm{b}} x_{0}=$ threshold expressed as number of infected fruit per sample (corresponding to a disease incidence of $0.5 \%$ ). In column $T_{l},-$ indicates sample size is too small to allow acceptance of load based on absence of infected fruit. In column $T_{u}$, - indicates sample size is greater than the maximum number of samples, $n_{\max }$, that need to be collected if the cumulative number of infected fruit stays between $T_{u}$ and $T_{l}$; and $n_{\max }$ calculated with precision $D=0.25$ (equation 4). 
sampling plan to total fruit number, an assessor would have to count all fruit, not only infected fruit, during assessment of the first few samples. Based on the results, the assessor would then select the appropriate column in Table 2 and continue sampling as suggested by the table, counting only infected fruit.

Resampling analysis. Resampling analyses were carried out to determine the number of samples an assessor using the sequential sampling plan would have to draw from each fruit load before deciding whether mummy berry incidence is above or below the threshold. The results showed that the mean and standard deviation of the average sample number at which the stop limit was reached was greatest when disease incidence was close to the $0.5 \%$ threshold and least for incidence levels $>1 \%$ (Fig. 4). In general, the average sample number required before a decision could be made was lower than $n_{\max }$. Indeed, most fruit loads could be classified reliably with $<10$ samples per load (Fig. 4). For loads with a disease incidence very close to the threshold, $<50$ samples were needed on average.

Theoretical and practical considerations related to sequential sampling. As stated previously, the main incentive in using Iwao's procedure $(9,10)$, an empirical, regression-based approach, for developing the sequential sampling plans presented here was the method's flexibility in adapting to variable dispersion patterns across fruit loads without the need to assume an underlying theoretical frequency distribution that fits all loads. In contrast, distribution-based approaches such as Wald's procedure generally require a single frequency distribution with fixed parameter values describing all populations that the sequential sampling plan is to be applied to; this requirement was not met in the present study (Table 1).

Because Iwao's procedure is not distribution-based, the approach cannot be validated by simulation from such a distribution. Instead, we employed computer-based resampling from packinghouse data sets to arrive at average performance values and associated variances. The relative merits of resampling compared with distribution-based methods for validating sampling plans have been discussed previously $(4,17)$. In general, resampling is most reliable with data sets that cover the range of population means

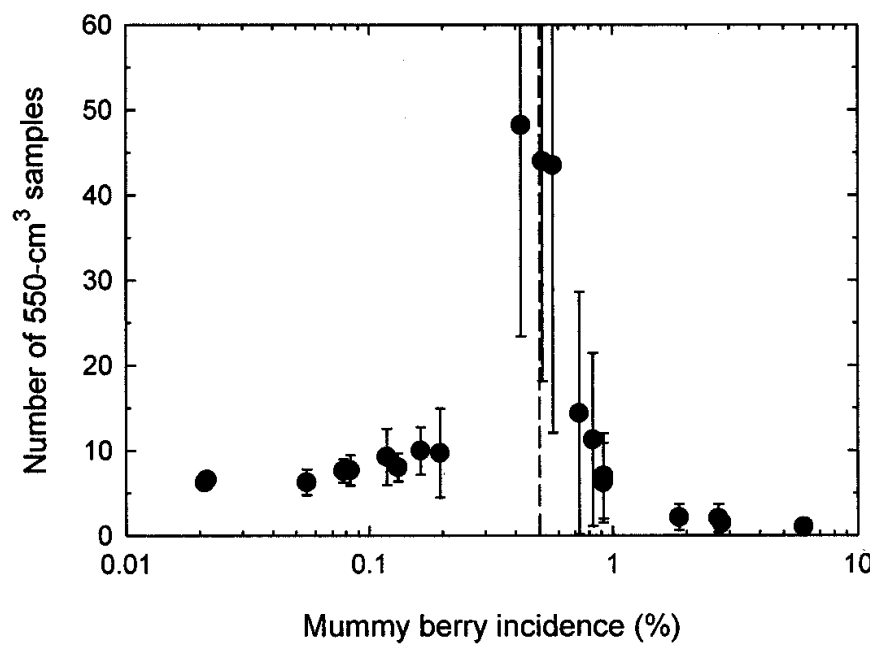

Fig. 4. Resampling analysis of the effect of the incidence of fruit infected by Monilinia vaccinii-corymbosi in loads of mechanically harvested rabbiteye blueberries from commercial packinghouses in southeastern Georgia on the average number of fruit samples needed to determine whether disease incidence is above or below a threshold of $0.5 \%$ (dashed vertical line). Simulations were done by randomly (without replacement) drawing samples from each load until one of the stop lines for sequential sampling (equations 2 and 3 ) or $n_{\max }$ (the maximum number of samples that needs to be collected if the cumulative number of infected fruit stays between the two stop lines) was reached; $n_{\max }$ calculated with precision $D=0.25$ (equation 4 ). Values are means and standard deviations of 500 computer runs per load. encountered in practice when a sufficiently large $(>20)$ number of data sets, each with a sufficiently large $(>50)$ number of samples, is available (4). These criteria were met in this study, particularly with the 1998 data sets. Indeed, the results suggested a high level of precision, with coefficients of variation $<0.05$ when average sample number for a given load was determined by independent 500-run resampling analyses (data not shown).

Because of the economic importance of mummy berry disease to growers, packinghouse operators, commodity buyers, and processors, a statistically reliable procedure is needed to quantify disease incidence under commercial conditions. It is hoped that the sequential sampling strategy derived in this study, in conjunction with the improved disease detection protocol developed previously (20), will meet this need.

\section{ACKNOWLEDGMENTS}

We thank J. Clark, D. Stanaland, M. Bruorton, and J. Smith, Extension Directors in Appling, Bacon, Clinch, and Pierce County, respectively, for assistance in communicating with packinghouse operators in southeastern Georgia and A. Ferrenberg, University of Georgia Research and Computational Science Support, for programming assistance.

\section{LITERATURE CITED}

1. Austin, M. E. 1994. Rabbiteye Blueberries. Development, Production and Marketing. Agscience Inc., Auburndale, FL.

2. Batra, L. R. 1983. Monilinia vaccinii-corymbosi (Sclerotiniaceae): Its biology on blueberry and comparison with related species. Mycologia 75:131-152.

3. Binns, M. R. 1994. Sequential sampling for classifying pest status. Pages 137-174 in: Handbook of Sampling Methods for Arthropods in Agriculture. L. P. Pedigo and G. D. Buntin, eds. CRC Press, Boca Raton, FL.

4. Binns, M. R., Nyrop, J. P., and van der Werf, W. 2000. Sampling and Monitoring in Crop Protection. CABI Publishing, Wallingford, U.K.

5. Boivin, G., Kushalappa, A. C., and Brodeur, L. 1990. Spatial dispersion and binomial sequential sampling plan for Cercospora carotae on carrots. Can. J. Plant Pathol. 12:209-212.

6. Campbell, C. L., and Madden, L. V. 1990. Introduction to Plant Disease Epidemiology. John Wiley \& Sons, New York.

7. Hart, L. P., and Schabenberger, O. 1998. Variability of vomitoxin in truckloads of wheat in a wheat scab epidemic year. Plant Dis. 82: 625-630.

8. Hildebrand, P. D., Milholland, R. D., and Stretch, A. W. 1995. Mummy berry. Pages 11-12 in: Compendium of Blueberry and Cranberry Diseases. F. L. Caruso and D. C. Ramsdell, eds. The American Phytopathological Society, St. Paul, MN.

9. Iwao, S. 1968. A new regression method for analyzing the aggregation pattern of animal populations. Res. Popul. Ecol. 10:1-20.

10. Iwao, S. 1975. A new method of sequential sampling to classify populations relative to a critical density. Res. Popul. Ecol. 16:281-288.

11. Karandinos, M. G. 1976. Optimum sample size and comments on some published formulae. Bull. Entomol. Soc. Am. 22:417-421.

12. Lockhart, C. L., Delbridge, R. W., and McIsaac, D. 1983. Observations on Monilinia twig and blossom blight of the lowbush blueberry in the Maritime Provinces. Can. Plant Dis. Surv. 63:31-34.

13. Madden, L. V. 1989. Dynamic nature of within-field disease and pathogen distributions. Pages 96-126 in: Spatial Components of Plant Disease Epidemics. M. J. Jeger, ed. Prentice Hall, Englewood Cliffs, NJ.

14. Madden, L. V., and Hughes, G. 1999. Sampling for plant disease incidence. Phytopathology 89:1088-1103.

15. Marois, J. J., Bledsoe, A. M., Ricker, R. W., and Bostock, R. M. 1993. Sampling for Botrytis cinerea in harvested grape berries. Am. J. Enol. Vitic. 44:261-265.

16. Naranjo, S. E., Diehl, J. W., and Ellsworth, P. C. 1997. Sampling whiteflies in cotton: Validation and analysis of enumerative and binomial plans. Environ. Entomol. 26:777-788.

17. Naranjo, S. E., and Hutchison, W. D. 1997. Validation of arthropod sampling plans using a resampling approach: Software and analysis. Am. Entomol. 43:48-57.

18. Pedigo, L. P., and Zeiss, M. R. 1996. Analyses in Insect Ecology and Management. Iowa State University Press, Ames.

19. Rouse, D. I., MacKenzie, D. R., Nelson, R. R., and Elliott, V. J. 1981. Distribution of wheat powdery mildew incidence in field plots and relationship to disease severity. Phytopathology 71:1015-1020.

20. Scherm, H., and Copes, W. E. 1999. Evaluation of methods to detect 
fruit infected by Monilinia vaccinii-corymbosi in mechanically harvested rabbiteye blueberry. Plant Dis. 83:799-805.

21. Scherm, H., Horton, D. L., NeSmith, D. S., Krewer, G., and Savelle, A. T. 1999. Georgia blueberry pest management survey. Pages 37-45 in: Blueberry Research at the University of Georgia: 1999 Annual Research Update. D. S. NeSmith, ed. Res. Rep. 662.

22. Shaw, P. B., Kido, H., Flaherty, D. L., Barnett, W. W., and Andris, H. L. 1983. Spatial distribution of infestations of Platynota stultana (Lepidoptera: Tortricidae) in California vineyards and a plan for sequential sampling. Environ. Entomol. 12:60-65.
23. Vincelli, P. C., and Lorbeer, J. W. 1987. Sequential sampling plan for timing initial fungicide application to control Botrytis leaf blight of onion. Phytopathology 77:1301-1303.

24. Wald, A. 1947. Sequential Analysis. John Wiley \& Sons, New York.

25. Waters, W. E. 1955. Sequential sampling in forest insect surveys. For. Sci. 1:68-79.

26. Wipfli, M. S., Peterson, S. S., Hogg, D. B., and Wedberg, J. L. 1992. Dispersion patterns and optimum sample size analyses for three plant bug (Heteroptera: Miridae) species associated with birdsfoot trefoil in Wisconsin. Environ. Entomol. 21:1248-1252. 